University of Nebraska - Lincoln

DigitalCommons@University of Nebraska - Lincoln

1995

\title{
Submaximal Oviposition Rates in a Mymarid Parasitoid: Choosiness Should Not Be Ignored
}

\author{
Amos Bouskila \\ Ben Gurion University of the Negev \\ Ian C. Robertson \\ Simon Fraser University \\ Michael E. Robinson \\ Simon Fraser University \\ Bernard D. Roitberg \\ Simon Fraser University \\ Brigitte Tenhumberg \\ University of Nebraska - Lincoln, btenhumberg2@unl.edu \\ See next page for additional authors
}

Follow this and additional works at: https://digitalcommons.unl.edu/bioscifacpub

Part of the Life Sciences Commons

\footnotetext{
Bouskila, Amos; Robertson, Ian C.; Robinson, Michael E.; Roitberg, Bernard D.; Tenhumberg, Brigitte; Tyre, Andrew J.; and vanRanden, Ed, "Submaximal Oviposition Rates in a Mymarid Parasitoid: Choosiness Should Not Be Ignored" (1995). Faculty Publications in the Biological Sciences. 207.

https://digitalcommons.unl.edu/bioscifacpub/207

This Article is brought to you for free and open access by the Papers in the Biological Sciences at DigitalCommons@University of Nebraska - Lincoln. It has been accepted for inclusion in Faculty Publications in the Biological Sciences by an authorized administrator of DigitalCommons@University of Nebraska - Lincoln.
} 


\section{Authors}

Amos Bouskila, lan C. Robertson, Michael E. Robinson, Bernard D. Roitberg, Brigitte Tenhumberg, Andrew J. Tyre, and Ed vanRanden 


\title{
Submaximal Oviposition Rates in a Mymarid Parasitoid: Choosiness Should Not Be Ignored
}

\author{
Amos Bouskila, ${ }^{1,2}$ Ian C. Robertson, ${ }^{1}$ Michael E. Robinson, ${ }^{1}$ Bernard D. Roitberg, ${ }^{1}$ \\ Brigitte Tenhumberg, ${ }^{1}$ Andrew J. Tyre, ${ }^{1}$ and Ed vanRanden ${ }^{1}$ \\ 1. Behavioural Ecology Research Group, Department of Biological Sciences, \\ Simon Fraser University, Burnaby, British Columbia, Canada V5A $1 S 6$. \\ 2. Present address: Department of Life Sciences, \\ Ben Gurion University of the Negev, 84105 Beer Sheva, Israel.
}

C ronin and Strong (1993a, b) examined the oviposition behavior of Anagarus delicatus Dozier (Hymenoptera: Mymaridae), an egg parasitoid of the leafhopper Prokelisia marginate Van Duzee (Homoptera: Delphacidae). They reported oviposition rates that were lower than predicted if the rate of egg-laying was maximized. Cronin and Strong (1993a) considered, and subsequently rejected, several "rules of thumb" (Stephens and Krebs 1986) as explanations for the observed patterns of patch departure. They observed that hosts on leaves experience density-independent mortality due to leaf senescence. Based on that, they advanced the hypothesis that submaximal oviposition rates in A. delicatus are best explained as risk-spreading by the parasitoid. (That is, by laying a small number of eggs on many leaves, the parasitoid increases the probability that some of her offspring will survive; Cronin and Strong 1993a.) An alternative hypothesis (Rosenheim and Mangel 1994) suggests that by distributing the eggs among several leaves, A. delicatus avoids self-superparasitism. Here, we take a somewhat different approach than those provided above to explain an additional observation of Cronin and Strong (1993a): female wasps rejected most of the hosts that they had probed. In so doing, we argue that rate maximization was an inappropriate prediction for A. delicatus, and in light of the life history parameters of this species, egg limitation is more suitable. Furthermore, egg limitation, when combined with one of the proposed explanations for the distribution of eggs, can explain the high rejection level of potential hosts.

Cronin and Strong found that $A$. delicatus has a limited egg load, ranging between 21 and 45 eggs $(\bar{X}=$ 33.3, Cronin and Strong 1990a: Table 1). They also ob- served that $A$. delicatus lives for up to $2 \mathrm{~d}$ and, in captivity, can oviposit $95 \%$ of its entire egg load in $8 \mathrm{~h}$. Moreover, $6 \%$ of 200 field-caught females had laid all of their eggs (as evidenced by empty ovarioles, Cronin 1991), suggesting that a large proportion of wasps would deplete their ovarioles by the end of their lifetime. We suggest that these animals appear to be egg-limited rather than time-limited. Because animals should only ratemaximize when limited by time, rate-maximization is unlikely to be appropriate for A. delicatus. Cronin and Strong (1993a) dismissed egg limitation because wasps left patches before depleting their egg supply. Although we agree that egg limitation does not explain the low number of eggs laid per patch, egg limitation predicts selectivity of hosts (e.g., Mangel and Roitberg 1989). Indeed, $A$. delicatus appears to be very selective because it probes $\approx 50 \%$ of potential hosts with its ovipositor (Cronin and Strong 1993b), but accepts only $8.4 \%$ (6 of 71 on average; Cronin and Strong 1993a).

Although Cronin and Strong acknowledge that $A$. delicatus may be choosy, they seem to dismiss selectivity by suggesting that all leafhopper eggs are suitable hosts. This was based on high parasitoid survival to the second instar, even when 20 wasps were induced to parasitize up to $90 \%$ of hosts in a patch (average number of hosts per patch was 131). Their suggestion ignores the possibility that variation in host quality could affect components of fitness other than survival to the second instar. If all hosts were acceptable, and if risk-spreading was the main cause of selection operating on patch departure behavior, as Cronin and Strong (1993a) imply, two predictions could be made: (a) wasps should oviposit some fixed number of hosts per patch (determined 
Table 1. Acceptance thresholds (minimum host quality that will be accepted) for selected conditions (rows) and eggloads (columns), and a single time period $(t=1)$. The first three rows are derived from a single run of the model, and show the effect of expected host distribution. Good hosts are more common in higher number worlds. The last six rows represent different runs, and show the effects of altering model parameter values (in world 3). " $m$ " is mortality per time unit; increase in the variable " $l$ " increases absolute differences in host value.

Acceptance threshold

\begin{tabular}{lllllllllllllllll} 
Conditions & \multicolumn{10}{c}{ Eggloads range from lowest $(e=1$, left side) to highest $(e=31$, right side $)$} \\
\hline World 1 & 6 & 5 & 5 & 5 & 5 & 5 & 5 & 5 & 4 & 4 & 4 & 4 & 4 & 4 & 4 & 4 \\
World 3 & 8 & 7 & 7 & 7 & 7 & 6 & 6 & 6 & 6 & 6 & 6 & 6 & 6 & 6 & 6 & 6 \\
World 5 & 9 & 9 & 9 & 8 & 8 & 8 & 8 & 8 & 8 & 8 & 8 & 7 & 7 & 7 & 7 & 7 \\
$m \times 0.5$ & 8 & 8 & 7 & 7 & 7 & 7 & 7 & 7 & 7 & 7 & 6 & 6 & 6 & 6 & 6 & 6 \\
$m \times 1.5$ & 8 & 7 & 7 & 6 & 6 & 6 & 6 & 6 & 6 & 6 & 6 & 5 & 5 & 5 & 5 & 5 \\
$m \times 2.0$ & 7 & 7 & 7 & 6 & 6 & 6 & 6 & 6 & 6 & 7 & 7 & 7 & 7 & 7 & 7 & 7 \\
$l=10$ & 7 & 7 & 7 & 6 & 6 & 6 & 6 & 6 & 6 & 6 & 6 & 6 & 6 & 5 & 5 & 5 \\
$l=20$ & 8 & 7 & 7 & 7 & 7 & 7 & 6 & 6 & 6 & 6 & 6 & 6 & 6 & 6 & 6 & 6 \\
$l=40$ & 8 & 7 & 7 & 7 & 7 & 7 & 6 & 6 & 6 & 6 & 6 & 6 & 6 & 6 & 6 & 6 \\
\hline
\end{tabular}

by the costs and benefits of moving between patches), and (b) wasps should not reject hosts after probing them. Only the first prediction was confirmed. Moreover, if life expectancy of ovipositing females declines over time, spending time probing hosts in a patch will be costly, and ovipositing the optimal number of eggs should be done as rapidly as possible. Host probing and rejection is only predicted if (a) variation in host quality exists, and if wasps are egg-limited to a degree that makes choosiness adaptive, or (b) "apparent" rejection occurs when wasps probe but are unable to complete oviposition because they fail to contact the host, contact host tissue unsuitable for oviposition, or some other random mechanism. Although Cronin and Strong (1993a, 1993b) observed that the wasps are apparently unable to detect previous parasitisms, it is possible that wasps can detect variation in host quality in spite of their inability to detect previous parasitisms.

The number of hosts parasitized by A. delicatus is independent of the number of hosts per patch (Stiling and Strong 1982, Cronin and Strong 1990b, 1993a). In order to explain selectivity given the density independence of parasitization, we suggest a simple "rule of thumb" that may be employed by an ovipositing female: within a patch, use the quality of the last few hosts probed to update the estimate of patch quality (similar to the sliding "memory window" of Li et al. 1993). Based on the estimate of patch quality and on the number of remaining eggs, determine the rejection threshold, and attack the next host above that threshold. The process is repeated until the optimal number of eggs to lay within a patch is reached. This optimal number must be determined by another process, such as risk-spreading (Cronin and Strong 1993a). Our hypothesis requires that choosiness is adaptive.

To illustrate the point, we developed a simple state variable model of parasitoid foraging on hosts of vari- ous qualities. As we do not seek to explain patch-leaving behavior, we make the simplifying assumption that wasps lay all their eggs on a single patch. There are 11 different host qualities, and the mean host quality in a patch is referred to as the patch quality. All patches have the same variance in host quality. Expected fitness is discounted by a small constant risk of death at each time unit. The first part of the model computes the optimal host rejection threshold for each combination of the two state variables, eggload and time, for a particular patch quality. The optimal behavior for each combination of state variables is computed by calculating the expected fitness of each possible host rejection threshold, and selecting the threshold that has the highest expected fitness. This is repeated for each different patch quality, creating a decision matrix (see Table 1) for each possible distribution of hosts (by backward induction, Mangel and Clark 1988). Our estimate of expected fitness for a particular time, eggload, and patch quality is:

$$
\begin{aligned}
& F(p, e, t, T) \\
& =\operatorname{MAX}_{\text {th }}\left\{\begin{array}{l}
F(p, e, t+1, T) \cdot(1-\mathrm{m}) \cdot \sum_{\text {type }=1}^{\text {th- } 1} f(p, \text { type }) \\
\begin{array}{l}
+\sum_{\text {type }=\text { th }} f(p, \text { type }) \cdot[v(\text { type }) \\
+(1-m) \cdot F(p, e-1, t+1, T)
\end{array}
\end{array}\right\}
\end{aligned}
$$

where $p$ is patch quality, $e$ is eggload, $t$ is the current time step, $T$ is the end of a wasp's life, $m$ is the probability of mortality in a single time step, "type" is the host type, "max type" is the highest quality host type, "th" is the host rejection threshold. " $v$ (type)" is the value of a host type to the parasitoid, and can be interpreted as the probability of an offspring surviving to adulthood on that host. " $f(p$, type $)$ " is the frequency of type in a patch of quality $p$. The operator MAX $_{\text {th }}$ represents maximiz- 


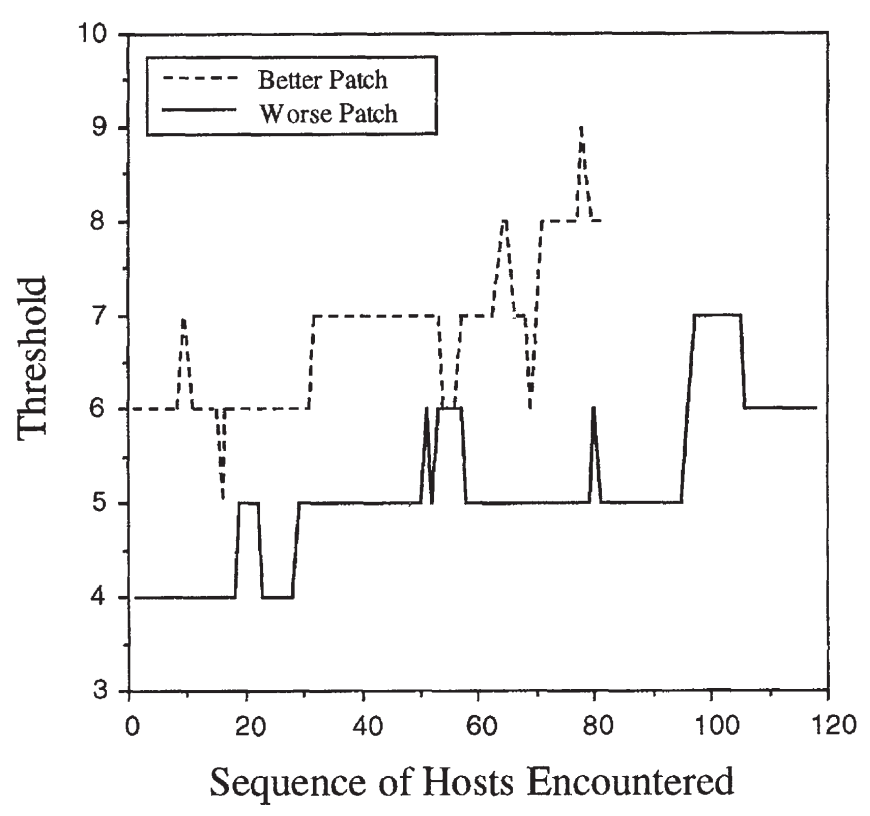

Figure 1. Host rejection thresholds of individual wasps as a function of time.

ing the equation over all possible values of th. The second part of the model simulates the behavior of individual wasps that encounter hosts stochastically. At each time unit the wasps use recent encounters with hosts to form an estimate of patch quality according to our "rule of thumb," and then parasitize or reject hosts based on the threshold drawn from the decision matrix for the estimated patch quality.

We can make three generalizations if we follow the host rejection thresholds of individual wasps throughout their simulated lifetimes (Figure 1). First, the host rejection threshold of an individual tends to increase with time. This occurs because wasps become more selective about which hosts they accept as their eggload declines. Second, hosts that were rejected at one point in time may be accepted later because the host rejection threshold decreases when, occasionally, wasps encounter several low-quality hosts in succession and thus assess their habitat as poor. This accounts for Cronin and Strong's (1993a) observation that wasps sometimes oviposited in eggs that had been probed and rejected previously. Third, wasps tend to lay all of their eggs in a shorter period of time in patches of higher quality.

We used the model to simulate several "experiments" to further test the "rule of thumb." In the first experiment, from 1 to 10 wasps oviposited simultaneously in a patch until each wasp had laid 6 eggs, and the percentage of the total number of hosts parasitized was recorded. In an additional experiment, 20 wasps were placed on a patch and forced to oviposit their entire egg-

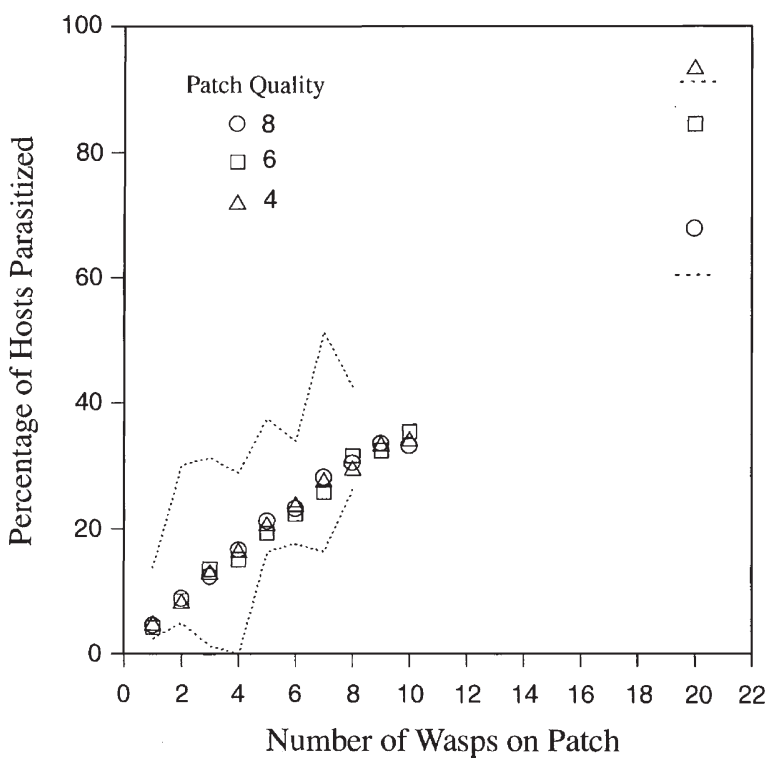

Figure 2. Percentage of hosts parasitized as a function of the number of wasps simultaneously present on a patch. The dotted lines represent minimum and maximum percentage parasitism estimated from data in Cronin and Strong (1993a: Figure 4).

load (32 eggs). These experiments mimic experimental procedures used by Cronin and Strong (1993a: Figure 4). The results of our experiments (Figure 2) are consistent with Cronin and Strong's results. The percentage of parasitized hosts increased with the number of wasps in a patch, reaching $68-93 \%$ when 20 wasps foraged simultaneously. Variation among different quality patches was large when 20 wasps foraged simultaneously, whereas variation among different quality patches for 1-10 wasps was small. Cronin and Strong used the results of their experiment to suggest that all hosts were acceptable to wasps. However, the model demonstrates that even if there are differences in quality among hosts, and wasps are choosy, the majority of hosts will be parasitized if the wasps are not given any other choices.

It is possible that rejection of hosts is not an adaptive behavior, but rather an apparent rejection caused by the wasp missing a host or contacting host tissue unsuitable for oviposition. In our second experiment we compared the fitness of wasps that followed the "rule of thumb" with the fitness of wasps that accepted and rejected hosts on a random basis, regardless of host quality. Such a protocol allowed us to determine if the "rule of thumb" is more adaptive than a random choice null model. The experiment showed that random choice results in lower fitness than the "rule of thumb." In a highquality patch (mean host quality $=8$ ) the fitness of randomly choosing females was $17.80 \pm 0.01$ (mean $\pm 1 \mathrm{sE}, n$ $=1000$ ), whereas selective females had a mean fitness of $18.44 \pm 0.01(n=1000)$. In a low-quality patch (mean host 
quality $=4)$, randomly choosing females had a mean fitness of $10.54 \pm 0.01(n=1000)$, whereas selective females had a mean fitness of $12.71 \pm 0.01(n=1000)$. Therefore, the "rule of thumb" yields an improvement in fitness over a random null model.

Although the model cannot eliminate the possibility that wasps are rejecting hosts randomly, we can suggest an experiment that would distinguish between the "rule of thumb" and the random rejection hypothesis. Present a sequence of wasps with the same patch of eggs. Each wasp is allowed to lay 6 eggs, and is then removed from the patch. Parasitized eggs are also removed. If wasps are rejecting hosts randomly, then their fitness would not depend on their position in the sequence, because the distribution of host qualities would not decline steadily with time. However, if wasps are using our "rule of thumb," we expect their fitness to decline with their position in the sequence because wasps earlier in the sequence preferentially remove the highest quality hosts. Fitness of females can be estimated by measuring survival of offspring to adulthood. When we simulate this experiment with our model, the fitness of wasps early in the sequence was nearly constant, with the last wasps experiencing a sharp reduction in fitness. This decline in fitness occurred earlier and was sharper for wasps in lower quality patches. In summary, we have suggested a "rule of thumb" for host acceptance decisions when there is variation in host quality and when average host quality in the patch is estimated from experience. A dynamic state variable model was used to formalize the predictions made from this "rule of thumb." Our model is very general and is applicable to any wasp species (e.g., Leptopilina heterotoma, Roitberg et al. 1992) wherein host acceptance is determined by the aforementioned parameters, but it can account for the patterns of host rejection observed by Cronin and Strong (1993b).

Acknowledgments - We thank J. T. Cronin, M. Mangel, and J. A. Rosenheim for stimulating discussions and D. Cohen, D. Strong, and anonymous reviewers for Ecology for suggestions on the manuscript. This work was supported by a Natural Sciences and Engineering Research Council of Canada Research Grant to B. D. Roitberg.

\section{Literature Cited}

Cronin, J. T. 1991. Parasitoid foraging behavior and the stabilization of host-parasitoid populations. Dissertation. Florida State University, Tallahassee, Florida, USA.

Cronin, J. T., and D. R. Strong. 1990a. Biology of Anagrus delicatus (Hymenoptera: Mymaridae), an egg parasitoid of Prokelisia marginate (Homoptera: Delphacidae). Annals of the Entomological Society of America 83:846-854.

Cronin, J. T., and D. R. Strong. 1990b. Density independent parasitism among host patches by Anagrus delicatus (Hymenoptera: Mymaridae): Experimental manipulation of hosts. Journal of Animal Ecology 59:1019-1026.

Cronin, J. T., and D. R. Strong. 1993a. Substantially submaximal oviposition rates by a mymarid egg parasitoid in the laboratory and field. Ecology 74:1813-1825.

Cronin, J. T., and D. R. Strong. 1993b. Superparasitism and mutual interference in the egg parasitoid Anagrus delicatus (Hymenoptera: Mymaridae). Ecological Entomology 18: 293-302.

Li, C., B. D. Roitberg, and M. Mackauer. 1993. Patch residence time and parasitism of Aphelinus asychis: A simulation model. Ecological Modelling 69:227-241.

Mangel, M., and C. W. Clark. 1988. Dynamic modeling in behavioral ecology. Princeton University Press, Princeton, New Jersey, USA.

Mangel, M., and B. D. Roitberg. 1989. Dynamic information and host acceptance by a tephritid fruit fly. Ecological Entomology 14:181-189.

Roitberg, B. M. Mangel, R. Lalonde, C. Roitberg, J. van Alphen, and L. Vet. 1992. Seasonal dynamic shifts in patch exploitation by parasitic wasps. Behavioral Ecology 3:156-165.

Rosenheim, J. A., and M. Mangel. 1994. Patch leaving rules for parasitoids with imperfect host discrimination. Ecological Entomology 19:374-380.

Stephens, D. W., and J. R. Krebs. 1986. Foraging theory. Princeton University Press, Princeton, New Jersey, USA.

Stiling, P. D., and D. R. Strong. (1982) Egg density and the intensity of parasitism in Prokelisia marginate (Homoptera: Delphacidae). Ecology 63:1630-1635. 